\title{
Testing the interpretation of the Fermi Galactic center excess in terms of unresolved point sources
}

\section{Christoph Weniger}

GRAPPA, University of Amsterdam, Science Park 904, 1098XH, Amsterdam, Netherlands

E-mail: c.weniger@uva.nl

\section{Richard Bartels}

GRAPPA, University of Amsterdam, Science Park 904, 1098XH, Amsterdam, Netherlands

E-mail: r.t.bartels@uva.nl

\section{Suraj Krishnamurthy}

GRAPPA, University of Amsterdam, Science Park 904, 1098XH, Amsterdam, Netherlands

E-mail: s.krishnamurthy@uva.nl

\begin{abstract}
We introduce a new method to analyse populations of near-threshold sources in gamma-ray data. The method is based on a wavelet transform of the gamma-ray sky, using Mexican hat wavelets. The statistics of peak significances in the wavelet transform provides information about the spatial distribution and luminosity function of near-threshold sources. We apply this method to gammaray data from the inner Galaxy, in order to test the millisecond pulsar interpretation of the Fermi Galactic center excess. We find evidence for the unresolved point sources causing the excess emission at the $>4 \sigma$ level. For plausible luminosity functions, this new component explains $100 \%$ of the observed excess emission.
\end{abstract}

The 34th International Cosmic Ray Conference,

30 July- 6 August, 2015

The Hague, The Netherlands

\footnotetext{
* Speaker.
} 


\section{Introduction}

Over the last years, various groups identified in the Fermi-LAT data an excess emission at $\mathrm{GeV}$ energies $[1,2,3,4,5,6,7,8,9,10,11]$, above the a priori expected astrophysical foreand backgrounds in that region, the Fermi 'Galactic center excess' (GCE). The excess features a remarkably uniform spectrum, an approximately spherical symmetry $[9,11]$, and it is detected from the inner 10 arcmin up to 10-20 deg latitude. Its physical cause remains a matter of debate. It turned out to be rather challenging to explain all of the GCE as effects of diffuse emission, like the proposed leptonic or hadronic outbursts from the Galactic center (GC) [12, 13, 14]. One of the most exciting possibilities is that the excess emission in the inner Galaxy is a signal from dark matter annihilation.

The probbaly most plausible astrophysical interpretation for the GCE is the combined emission from a large number of unresolved point sources, with the best candidates being millisecond pulsars (MSPs) $[6,8,15,16]$. Indeed, MSPs where found to feature a stacked gamma-ray spectrum that is compatible with the GCE emission [11]. However, it was claimed that MSPs cannot account for $100 \%$ of the observed GCE: A significant fraction of the necessary MSPs population should have already been seen by the Fermi-LAT $[17,18]$ as resolved sources. However, this conclusion depends crucially on the maximal $\gamma$-ray luminosity of the MSP population, and on details of the Fermi-LAT sensitivity [19]. A reliable sensitivity study was lacking up to now.

Here, summarizing results from Ref. [20], we present a new method for the analysis of dim gamma-ray source populations. We use it to search for contributions from a large number of dim MSP-like sources, assuming a that this population is spatially distributed like suggested by the GCE.

\section{Method}

We simulate a population of MSP-like sources, which we henceforth simply denote as central source population (CSP). We assume that the spatial distribution follows a radial power-law with an index of $\Gamma=-2.5$ and a cutoff at radius $r=3 \mathrm{kpc}[9,11]$. We adopt the stacked MSP spectrum from Ref. [21], $\frac{d N}{d E} \propto e^{-E / 3.78 \mathrm{GeV}} E^{-1.57}$ as reference energy spectrum. The luminosity function $\frac{d N}{d L}$ is modeled by a power-law with index $\alpha=-1.5[21,19]$. It is non-zero within the $\gamma$-ray luminosity range $L_{\min }=10^{31} \mathrm{erg} \mathrm{s}^{-1}$ and $L_{\max }=10^{34}-10^{36} \mathrm{erg} \mathrm{s}^{-1}$, and zero otherwise. Gammaray luminosities are integrated over $0.1-100 \mathrm{GeV}$. Consistency of the CSP gamma-ray luminosity function with the luminosities of nearby resolved MSPs requires a maximum luminosity of least $L_{\max } \gtrsim 1.0 \cdot 10^{35} \mathrm{erg} \mathrm{s}^{-1}$. As model for the Galactic diffuse emission, we adopt a standard diffuse model and the isotropic backgrounds, although our results do not depend critically on this choice.

We used five years of reprocessed clean Fermi-LAT P7 data, from August 2008 to September 2013 (both front and back converted events). We produce count maps for the energy range 1-4 $\mathrm{GeV}$, and concentrate on the 'inner Galaxy' Region Of Interest (ROI), which spans $|\ell| \leq 12^{\circ}$ and $2^{\circ} \leq|b| \leq 12^{\circ}$.

Our analysis is based on the wavelet transform, which is defined as the convolution of the photon count map $\mathscr{C}(\Omega)$ with the wavelet kernel $\mathscr{W}\left(\Omega^{\prime}\right), \mathscr{F} \mathscr{W}[\mathscr{C}](\Omega) \equiv \int d \Omega \mathscr{W}\left(\Omega-\Omega^{\prime}\right) \mathscr{C}\left(\Omega^{\prime}\right)$, 


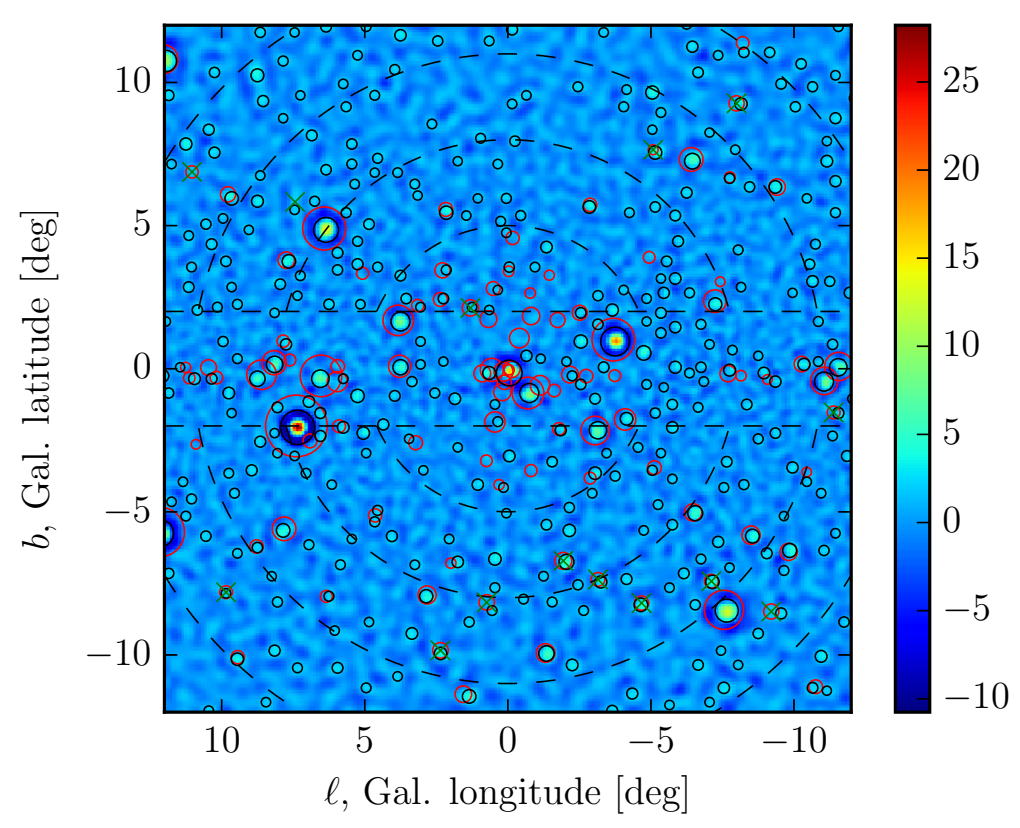

Figure 1: SNR of the wavelet transform of $1-4 \mathrm{GeV} \gamma$-rays, $\mathscr{S}(\Omega)$, with values as indicated in the color bar. Black (red) circles show the position of wavelet peaks (3FGL sources) with statistical significance $\mathscr{S} \geq 2$ $(\sqrt{T S} \geq 2)$; the covered area scales with the significance. The dashed black lines indicate the regions that we use in the statistical analysis. The inner $|b|<2^{\circ}$ are excluded because the strong emission from the Galactic disk leads to spurious sources. Reproduction from Ref. [20].

where $\Omega$ denotes Galactic coordinates. In our analysis, we will actually focus on the signal-to-noise ratio (SNR), which we define as

$$
\mathscr{S}(\Omega)=\frac{\mathscr{F}_{\mathscr{W}}[\mathscr{C}](\Omega)}{\sqrt{\mathscr{F}_{\mathscr{W}^{2}}[\mathscr{C}](\Omega)}}
$$

In order to get an estimate for the level of background noise, in the denominator the wavelet kernel is squared before performing the convolution. We adopt throughout the second member of the Mexican Hat Wavelet Family, MHWF2 [22]. The wavelet width is given by $\sigma_{b} \cdot R$, where $\sigma_{b}=0.4^{\circ}$ indicates the Fermi-LAT angular resolution at $1-4 \mathrm{GeV}$, and $R$ is a tuning parameter. We let $R$ linearly vary with latitude, from $R=0.55$ at $b=0^{\circ}$ to $R=0.91$ at $b= \pm 12^{\circ}$, which compensates the increasing variability of backgrounds towards the Galactic disk [23].

In Fig. 1 we show the resulting SNR of the wavelet transform of inner Galaxy ROI. The Galactic diffuse emission almost completely disappeared thanks to the wavelet transform, but the bright gamma-ray sources lead to pronounced peaks. We also show the identified peaks in the wavelet transform, with SNR above two, $\mathscr{S}>2$. These are compared with the 3FGL sources (detected in the energy band $1-3 \mathrm{GeV}$ with $\sqrt{T S} \geq 2$ ) [24]. In general, we find at high latitudes a good correspondence between wavelet peaks and 3FGL sources.

We mask almost all peaks that are closer to 3FGL sources than $0.3^{\circ}$. However, we keep peaks that are potentially part of the CSP, which we identify by requiring that they (i) are tagged as unassociated, (ii) show no indication for variability and (iii) have a spectrum compatible with 


\begin{tabular}{cccccc}
\hline 3FGL Name & $\ell\left[^{\circ}\right]$ & $b\left[^{\circ}\right]$ & $\chi^{2} /$ dof & $\sqrt{T S}$ & $\mathscr{S}$ \\
\hline $\mathrm{J} 1649.6-3007$ & -7.99 & 9.27 & 1.07 & 5.57 & 3.68 \\
$\mathrm{~J} 1703.6-2850$ & -5.08 & 7.65 & 0.48 & 2.38 & 4.24 \\
$\mathrm{~J} 1740.5-2642$ & 1.30 & 2.12 & 0.37 & 6.37 & 2.15 \\
$\mathrm{~J} 1740.8-1933$ & 7.43 & 5.83 & 0.77 & 1.89 & 2.11 \\
$\mathrm{~J} 1744.8-1557$ & 11.03 & 6.88 & 0.40 & 3.69 & 1.96 \\
$\mathrm{~J} 1758.8-4108$ & -9.21 & -8.48 & 0.90 & 5.56 & 2.91 \\
$\mathrm{~J} 1759.2-3848$ & -7.11 & -7.43 & 0.35 & 4.64 & 4.36 \\
$\mathrm{~J} 1808.3-3357$ & -1.94 & -6.71 & 0.40 & 6.94 & 5.46 \\
$\mathrm{~J} 1808.4-3519$ & -3.15 & -7.36 & 0.41 & 4.55 & 3.51 \\
$\mathrm{~J} 1808.4-3703$ & -4.68 & -8.19 & 0.22 & 4.95 & 4.45 \\
$\mathrm{~J} 1820.4-3217$ & 0.74 & -8.17 & 1.04 & 5.74 & 2.32 \\
$\mathrm{~J} 1830.8-3136$ & 2.35 & -9.84 & 0.54 & 5.92 & 3.76 \\
$\mathrm{~J} 1837.3-2403$ & 9.85 & -7.81 & 0.28 & 4.03 & 2.16 \\
\hline
\end{tabular}

Table 1: List of the 13 unassociated 3FGL sources with MSP-like spectra. It is likely that most of them are part of the CSP. The three last columns show the goodness-of-fit of the reference MSP spectrum, the 3FGL significance in the $1-3 \mathrm{GeV}$ band, and the corresponding peak of the wavelet SNR. Reproduction from Ref. [20].

MSPs. For the last criterion, we require that a fit of the above MSP reference spectrum to the spectrum given in the 3FGL $\left(0.1-100 \mathrm{GeV}\right.$; five energy bins) gives $\chi^{2} / \mathrm{dof} \leq 1.22$ (with dof $=4$ ). In this way, we identify 13 MSP candidate sources in the 3FGL in the inner Galaxy ROI, which are listed in Tab. 1. For comparison, the average number of MSP candidate sources in similar control regions along the Galactic disk is found to be significantly smaller, namely $3.1 \pm 1.5$.

\section{Statistical analysis and results}

In order to perform a statistical analysis of the peaks, we bin the peaks in a two dimensional grid. This covers the projected Galacto-centric angles $2^{\circ}-17^{\circ}$ (with regions as indicated in Fig. 1) as well as the SNR peak significances in the range 1-10. The number of peaks in each bin follows a Poisson distribution. It is hence enough to derive the corresponding expectation values with a Monte Carlo. The relevant free parameters are here the maximal luminosity, $L_{\max }$, as well as on the number of simulated sources, $n$. In order to allow comparison with previous work, we determine the number of sources $n$ in terms of the mean differential intensity of the CSP at latitudes of $b= \pm 5^{\circ}$, $\ell=0^{\circ}$ and energies of $2 \mathrm{GeV}$.

In Fig. 2 we show the resulting limits that we obtain on the CSP as function of the maximum luminosity $L_{\max }$ and the total differential intensity at $b= \pm 5^{\circ}, \Phi_{5}$. A non-zero contribution from the CSP is favoured with at least $4 \sigma$, depending on the assumptions about background sources. When performing the same analysis in six control regions along the Galactic disk (centered at $\ell= \pm 40$ $80^{\circ}$ ), we find instead null detections, as expected. The preferred range of the maximum gamma-ray luminosity is compatible with observations of MSPs, which is illustrated in Fig. 2 by the vertical lines (showing the gamma-ray luminosity of nearby MSPs from Ref. [21]). Furthermore, for the adopted slope of the luminosity function, $\alpha=1.5$, (and $L_{\min }=10^{31} \mathrm{erg} \mathrm{s}^{-1}$ ), the overall combined emission is consistent with producing $100 \%$ of the GCE emission. 


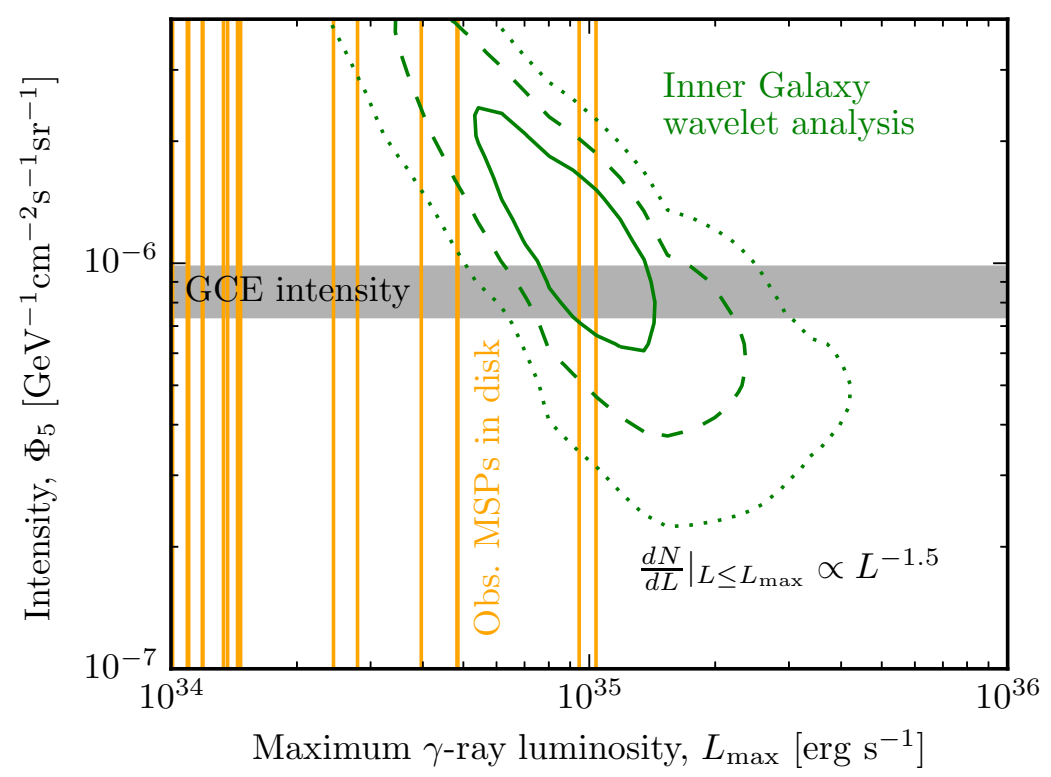

Figure 2: Constraints on the maximum $\gamma$-ray luminosity of the CSP, $L_{\max }$, and the population averaged intensity at $b= \pm 5^{\circ}, \ell=0^{\circ}$ and $2 \mathrm{GeV}, \Phi_{5}$, as derived from our wavelet analysis. We show 68.7\%, 95.4\% and $99.7 \% \mathrm{CL}$ bands. We also indicate the values of $\Phi_{5}$ where the source population can explain $100 \%$ of the GCE (horizontal gray band, 95.4\% CL), and as vertical orange lines the luminosity of the brightest observed nearby MSPs. Reproduction from Ref. [20].

\section{Conclusions}

We find strong support for the interpretation of the GCE in terms of a hitherto undetected population of MSP-like sources. The only assumption is that these sources are spatially distributed like suggested by the observed GCE (spherically symmetric, following an inverse radial powerlaw). From the inner Galaxy obseravtions, we obtain constraints on the maximum luminosity $L_{\max }$ and $\alpha$ that are in agreement with observations of nearby MSPs, and strongly supporting the interpretation in terms of MSPs. Although this is quite compelling, a first necessary step to fully establish this interpretation will be a confirmation that most of the MSP candidate sources in Tab. 1 can indeed be identified with MSPs within the inner $3 \mathrm{kpc}$ of the GC.

Acknowledgments. CW would like to thank for useful discussions with Francesca Calore, Fiorenza Donato, Mattia Di Mauro and Jason Hessels. SK and CW (P.I.) are part of the VIDI research programme "Probing the Genesis of Dark Matter", which is financed by the Netherlands Organisation for Scientific Research (NWO).

\section{References}

[1] L. Goodenough and D. Hooper, arXiv:0910.2998 [hep-ph].

[2] V. Vitale et al. [Fermi-LAT Collaboration], arXiv:0912.3828 [astro-ph.HE].

[3] D. Hooper and L. Goodenough, Phys. Lett. B 697 (2011) 412 [arXiv:1010.2752 [hep-ph]]. 
[4] D. Hooper and T. Linden, Phys. Rev. D 84 (2011) 123005 [arXiv:1110.0006 [astro-ph.HE]].

[5] K. N. Abazajian and M. Kaplinghat, Phys. Rev. D 86 (2012) 083511 [Phys. Rev. D 87 (2013) 129902] [arXiv:1207.6047 [astro-ph.HE]].

[6] C. Gordon and O. Macias, Phys. Rev. D 88 (2013) 8, 083521 [Phys. Rev. D 89 (2014) 4, 049901] [arXiv:1306.5725 [astro-ph.HE]].

[7] O. Macias and C. Gordon, Phys. Rev. D 89 (2014) 6, 063515 [arXiv:1312.6671 [astro-ph.HE]].

[8] K. N. Abazajian, N. Canac, S. Horiuchi and M. Kaplinghat, Phys. Rev. D 90 (2014) 2, 023526 [arXiv:1402.4090 [astro-ph.HE]].

[9] T. Daylan, D. P. Finkbeiner, D. Hooper, T. Linden, S. K. N. Portillo, N. L. Rodd and T. R. Slatyer, arXiv:1402.6703 [astro-ph.HE].

[10] B. Zhou, Y. F. Liang, X. Huang, X. Li, Y. Z. Fan, L. Feng and J. Chang, Phys. Rev. D 91 (2015) 12, 123010 [arXiv:1406.6948 [astro-ph.HE]].

[11] F. Calore, I. Cholis and C. Weniger, JCAP 1503 (2015) 038 [arXiv:1409.0042 [astro-ph.CO]].

[12] E. Carlson and S. Profumo, Phys. Rev. D 90 (2014) 2, 023015 [arXiv:1405.7685 [astro-ph.HE]].

[13] J. Petrovic, P. D. Serpico and G. Zaharijas, JCAP 1410 (2014) 10, 052 [arXiv:1405.7928 [astro-ph.HE]].

[14] I. Cholis, C. Evoli, F. Calore, T. Linden, C. Weniger and D. Hooper, arXiv:1506.05119 [astro-ph.HE].

[15] K. N. Abazajian, JCAP 1103 (2011) 010 [arXiv:1011.4275 [astro-ph.HE]].

[16] Q. Yuan and B. Zhang, JHEAp 3-4 (2014) 1 [arXiv:1404.2318 [astro-ph.HE]].

[17] D. Hooper, I. Cholis, T. Linden, J. Siegal-Gaskins and T. Slatyer, Phys. Rev. D 88 (2013) 083009 [arXiv:1305.0830 [astro-ph.HE]].

[18] I. Cholis, D. Hooper and T. Linden, JCAP 1506 (2015) 06, 043 [arXiv:1407.5625 [astro-ph.HE]].

[19] J. PetroviÄĞ, P. D. Serpico and G. Zaharijas, JCAP 1502 (2015) 02, 023 [arXiv:1411.2980 [astro-ph.HE]].

[20] R. Bartels, S. Krishnamurthy and C. Weniger, arXiv:1506.05104 [astro-ph.HE].

[21] I. Cholis, D. Hooper and T. Linden, arXiv:1407.5583 [astro-ph.HE].

[22] J. Gonzalez-Nuevo, F. Argueso, M. Lopez-Caniego, L. Toffolatti, J. L. Sanz, P. Vielva and D. Herranz, Mon. Not. Roy. Astron. Soc. 369 (2006) 1603 [astro-ph/0604376].

[23] P. A. R. Ade et al. [Planck Collaboration], Astron. Astrophys. 571 (2014) A28 [arXiv:1303.5088 [astro-ph.CO]].

[24] [Fermi-LAT Collaboration], arXiv:1501.02003 [astro-ph.HE]. 